\title{
Pacific
}

Journal of

Mathematics

\section{A HOMOTOPY TRANSFER FOR FINITE GROUP ACTIONS}

\author{
WILLIAM J. RALPH
}




\title{
A HOMOTOPY TRANSFER FOR FINITE GROUP ACTIONS
}

\author{
Bill RALPH
}

\begin{abstract}
We obtain a transfer for group actions on spaces for which the orbit map admits a section. This transfer exists for sets of homotopy classes as well as for any generalized homology theory.
\end{abstract}

Introduction. Intuitively, one feels that there should exist special relationships between the homotopy invariants of a space $Y$ and its quotient by the action of some finite group $G$. The main result of this paper is the construction, for an arbitrary homology theory, of a version of the transfer that exists for ordinary homology. Recall that this is a homomorphism $\tau: H_{n}(Y / G) \rightarrow H_{n}(Y)$ with the following properties:

(a) $\tau \circ \rho(z)=\sum_{g \in G} g * z$ for all $z \in H_{n}(Y)$,

(b) $\rho \circ \tau(v)=|G| v$ for all $v \in H_{n}(Y / G)$

where $\rho: H_{n}(Y) \rightarrow H_{n}(Y / G)$ is the projection. An account of this can be found in $[\mathrm{Br}]$.

Unfortunately, the existence of a transfer map satisfying (a) and (b), or their duals in cohomology, seems to be a special property of the ordinary homology and cohomology functors which is closely tied to the fact that Eilenberg-Mac Lane spaces have the homotopy type of abelian monoids. In view of this, it is not surprising that in general there is no transfer for covariant functors $F$ such as $\pi_{n}$ and those associated with generalized homology theories.

In this paper we will recover a version of transfer for many functors $F$ including generalized homology theories. In order to deal with the fact that the $H$-spaces that arise are not, in general, of the homotopy type of abelian monoids, we will have to multiply equation (a) by a number $c(G)$, that I have been calling the coherence number of the group $G$. This number depends only on the group $G$ and is currently under intense investigation. For cyclic groups this number is 1 and hence the transfer equations will have their usual form in this case. It is not yet known whether this number is always finite, so there may be groups to which our transfer cannot be applied, although our feeling 
is that this is not the case. [R3] contains all the current information on this number. The reason the coherence number is not required for abelian monoids can be understood in general form [R1] and, for ordinary homology in particular, from [R2].

1. Statement of results. Throughout this paper, we will assume that all spaces have basepoints, that $G$ is a finite group acting on a space $Y$, that $G$ fixes the basepoint of $Y$ and that the orbit map admits a section. We will let $\rho: Y \rightarrow Y / G$ denote the orbit map and let $s: Y / G \rightarrow Y$ denote a section of it. The symbol $c(G)$ which occurs in the following theorems denotes a positive integer or infinity and is a combinatorial invariant of the group that will be defined later.

The main results of this paper are the following:

THeORem 1. Let $Y$ have the homotopy type of a G-C.W. complex and suppose that $c(G)<\infty$. Let $\tilde{h}_{*}$ be the (reduced) homology theory associated with some spectrum and let $\rho: \tilde{h}_{n}(Y) \rightarrow \tilde{h}_{n}(Y / G)$ denote the obvious homomorphism. Then there exists a transfer homomorphism $\tau: \tilde{h}_{n}(Y / G) \rightarrow \tilde{h}_{n}(Y)$ satisfying the following:

(1) $c(G)\left(\tau \circ \rho(z)-\sum_{g \in G} g * z\right)=0$ for all $z \in \tilde{h}_{n}(Y)$,

(2) $\rho \circ \tau(v)=|G| v$ for all $v \in \tilde{h}_{n}(Y / G)$.

We also give a version of Theorem 1 for homotopy. See Theorem 6 below.

The following theorems give the flavour of the kind of constraints imposed by the transfer.

THEOREM 2. Suppose that $c(G)<\infty$ and there is some homology theory such that $\tilde{h}_{n}(Y)=\mathbb{Z}$ and $\tilde{h}_{n}(Y / G)=0$. Then $G$ must have a subgroup of index 2.

THEOREM 3. Suppose that $\mathbb{Z}_{4}$ acts on $W$ and induces an effective action on $\tilde{h}_{n}(W) \cong \mathbb{Z}_{16}$ for some homology theory. Then the orbit map $W \rightarrow W / \mathbb{Z}_{4}$ does not admit a section.

Here is the definition of the transfer map in its most general setting.

Definition 1. Let $X$ and $Y$ be spaces. Let $W$ be an $H$-space for which there exists a topological group $(L, 1)$ and a map $\varepsilon:\left(W, w_{0}\right) \rightarrow$ $(L, 1)$ inducing an isomorphism of groups $\bar{\varepsilon}:[X, W] \rightarrow[X, L]$. Let $\alpha$ be a map from $Y$ to $W$ and let $\bar{\alpha}$ be the induced map from $[X, Y]$ to $[X, W]$. Let $\bar{\rho}:[X, Y] \rightarrow[X, Y / G]$ be the map induced 
by the projection, let $\bar{s}:[X, Y / G] \rightarrow[X, Y]$ be the map induced by the section and let $\beta:[X, W] \rightarrow[X, W]^{\text {ab }}$ be the abelianizing map. The transfer map is the function

$$
\tau_{\alpha}:[X, Y / G] \rightarrow[X, W]^{\mathrm{ab}}
$$

defined by

$$
\tau_{\alpha}(v)=\sum_{g \in G} \beta \circ \bar{\alpha}(g * \bar{s}(v)) .
$$

2. Coherence numbers. As mentioned in the introduction, the development of this transfer requires a new idea, namely the "Coherence Number" of a finite group. We first define the coherence number of a set of permutations on a finite set and then specialize this definition to finite groups. The reader should refer to [R3] for details and proofs. Let $P(S)$ denote the set of all permutations on the set $S$.

Definition 2. Let $S$ be a finite set and let $A \subseteq P(S)$. Suppose that $S=\left\{s_{1}, \ldots, s_{m}\right\}$ and $A=\left\{\sigma_{1}, \ldots, \sigma_{n}\right\}$. Let $r_{i}=\left(\sigma_{1}\left(s_{i}\right), \ldots\right.$, $\left.\sigma_{n}\left(s_{i}\right)\right)$ and $c_{i}=\left(s_{i}, \ldots, s_{i}\right)$, for $i=1,2, \ldots, m$, be regarded as elements of $F(S)^{n}$, the direct product of $n$ copies of the free group on the set $S$. Let $T$ be the subgroup of $F(S)^{n}$ generated by $r_{1}, \ldots, r_{m}$ and $c_{1}, \ldots, c_{m}$ and define $\theta=\prod_{i=1}^{m} r_{i} c_{i}^{-1} \in T$. There is a homomorphism $\lambda: T \rightarrow T /[T, T]$, where $[T, T]$ is the commutator subgroup of $T$. The coherence number, $c(A)$, of $A$ is defined to be the order of $\lambda(\theta)$, which may be infinite. It is easily verified that $c(A)$ is independent of the ways in which $S$ and $A$ are ordered.

We now specialize this definition to groups by letting the group act on itself as a set of permutations.

Definition 3. Let $G$ be any finite group. We can regard $G$ as a subset of $P(G)$ by letting $G$ act on itself on the left by translation. Specifically, for $g \in G$, we define $\bar{g} \in P(G)$ by putting $\bar{g}(h)=$ $g h$. The coherence number of $\{\bar{g} \mid g \in G\} \subseteq P(G)$ will be called the coherence number of the group $G$ and will be denoted by $c(G)$. We will use $T_{G}$ to denote the subgroup corresponding to $T$ in Definition 2.

In [R3] we showed that the coherence numbers obtained by letting $G$ act on itself on the right or the left are the same. We have chosen Definition 3 as the most convenient in this context.

3. The combinatorial part of the transfer. In what follows we shall denote the action of $G$ on a point, function, homotopy class, etc. by $\mathrm{a} *$ and let the meaning be determined from the context. 
Definition 4. Let $X$ and $Y$ be sets and let $\left\{A_{i}\right\}$ be a set of $n$ subsets that cover $X$. If $f_{i}: X \rightarrow Y$ and $g_{l}: X \rightarrow Y$ for $i=1, \ldots, n$ are two families of maps, then we will say that $\left(f_{1}, \ldots, f_{n}\right) \sim\left(g_{1}, \ldots\right.$, $\left.g_{n}\right)$ if the restrictions of $f_{i}$ and $g_{l}$ to $A_{i}$ are the same for all $i=$ $1, \ldots, n$.

Our next lemma contains the essential combinatorial idea that makes the transfer work. Although it is only stated in terms of continuous functions, the identical proof works for arbitrary functions. In the following lemma, $(L, 1)$ is a topological group and $\zeta:\left(Y, y_{0}\right) \rightarrow$ $(L, 1)$ is a map. $\operatorname{Hom}\left(\left(X, x_{0}\right),\left(Y, y_{0}\right)\right)$ will denote the set of maps from $\left(X, x_{0}\right)$ to $\left(Y, y_{0}\right)$ and $\bar{\zeta}$ denotes the induced map from $\operatorname{Hom}\left(\left(X, x_{0}\right),\left(Y, y_{0}\right)\right)$ to $\operatorname{Hom}\left(\left(X, x_{0}\right),(L, 1)\right)$. We will regard $\operatorname{Hom}\left(\left(X, x_{0}\right),(L, 1)\right)$ as a group under pointwise multiplication.

LEMMA 1. Let $G$ be a finite group with finite group with finite coherence number. Let $\left(X, x_{0}\right)$ be a Hausdorff space and let $\left(Y, y_{0}\right)$ be a space on which $G$ acts. Let $\sigma, \omega \in \operatorname{Hom}\left(\left(X, x_{0}\right),\left(Y, y_{0}\right)\right)$ with $\rho \circ \sigma=\rho \circ \omega$. Let $M$ be the subgroup of $\operatorname{Hom}\left(\left(X, x_{0}\right),(L, 1)\right)$ generated by all elements of the form $\zeta \circ(g * \sigma)$ and $\zeta \circ(g * \omega)$, where $g$ is any element of $G$. Let $\delta: M \rightarrow M^{\mathrm{ab}}$ denote the projection. Then we have

$$
c(G) \sum_{g \in G} \delta \circ \bar{\zeta}(g * \sigma)=c(G) \sum_{g \in G} \delta \circ \bar{\zeta}(g * \omega)
$$

in $M^{\mathrm{ab}}$.

Proof. Recall, from Definitions (3) and (4), that the group $T_{G}$ is generated by the elements $r_{i}=\left(g_{i} g_{1}, \ldots, g_{i} g_{n}\right)$ and $c_{i}=\left(g_{i}, \ldots, g_{i}\right)$ for $i=1, \ldots, n$. The first step in our proof is to show that the homomorphism from $T_{G}$ into $\operatorname{Hom}\left(\left(X, x_{0}\right),(L, 1)\right)$ given on generators by $r_{i} \mapsto \zeta \circ\left(g_{i} * \sigma\right)$ and $c_{i} \mapsto \zeta \circ\left(g_{i} * \omega\right)$ is well defined.

Let $A_{i}=\left\{x \in X \mid \sigma(x)=g_{i} * \omega(x)\right\}$. Note that, since $\rho \circ \sigma=\rho \circ \omega$, these sets cover $X$. It is clear from the definition of the $A_{i}$ and Definition 4 that $(\sigma, \ldots, \sigma) \sim\left(g_{1} * \omega, \ldots, g_{n} * \omega\right)$. From this, we see in general that $(g * \sigma, \ldots, g * \sigma) \sim\left(\left(g g_{1}\right) * \omega, \ldots,\left(g g_{n}\right) * \omega\right)$.

We will construct a homomorphism $\psi_{x, k}: T_{G} \rightarrow L$ as follows. Let $\rho_{k}$ be the projection of the subgroup $T_{G}$ of $F(G)^{|G|}$ onto the $k$ th factor. For each $x \in X$, we define a map of sets, $\eta_{x}: G \rightarrow L$, by $\eta_{x}(g)=\zeta \circ(g * \omega)(x)$, which then induces a homomorphism $\bar{\eta}_{x}: F(G) \rightarrow F(L)$. Let $v: F(L) \rightarrow L$ be the canonical homomorphism and define $\psi_{x, k}=v \circ \bar{\eta}_{x} \circ \rho_{k}$. 
Now suppose that $x \in A_{j} \cap A_{k}$. We claim that $\psi_{x, j}=\psi_{x, k}$. To see this, the reader can easily check that equality holds on the two types of generators for the group $T_{G}$.

Since the sets $A_{k}$ cover the set $X$, it follows that the homomorphisms $\psi_{x, k}$, obtained from each pair $x$ and $k$ with $x \in A_{k}$, can be "glued" together into a single homomorphism $\psi$ from $T_{G}$ into the set of continuous functions from $\left(X, x_{0}\right)$ into $(L, 1)$. It is immediate from our construction of $\psi$ that $\psi\left(r_{i}\right)=\zeta \circ\left(g_{i} * \sigma\right)$ and $\psi\left(c_{i}\right)=\zeta \circ\left(g_{i} * \omega\right)$ and hence we obtain a well-defined map from $T_{G}$ into $\operatorname{Hom}\left(\left(X, x_{0}\right),(L, 1)\right)$.

Clearly the image of $\psi$ is precisely the subgroup $M$ of $\operatorname{Hom}\left(\left(X, x_{0}\right),(L, 1)\right)$ and so $\psi$ induces a homomorphism $\psi^{\mathrm{ab}}: T_{G}^{\mathrm{ab}} \rightarrow$ $M^{\text {ab }}$. Since the coherence number of $G$ was assumed to be finite, we know that $c(G) \lambda(\theta)=0$, where $\lambda(\theta)$ is the element of $T_{G}^{\mathrm{ab}}$ defined in Definition 2. Applying $\psi^{\mathrm{ab}}$, we see that $c(G) \psi^{\mathrm{ab}}(\lambda(\theta))=0$. Expanding this, we obtain that

$$
\begin{aligned}
c(G) \psi^{\mathrm{ab}}\left(\lambda\left(\prod_{i=1}^{m} r_{i} c_{i}^{-1}\right)\right) \\
=\left(c(G) \sum_{g \in G} \delta \circ \bar{\zeta}(g * \sigma)\right)-\left(c(G) \sum_{g \in G} \delta \circ \bar{\zeta}(g * \omega)\right)
\end{aligned}
$$

which equals zero and gives our result.

The reader should check that, if the topological group $L$ used above happened to be an abelian monoid, then we would not need the factor $c(G)$.

4. Properties of the transfer map. Here is the crucial property of the transfer map.

THEOREM 4. If $\tau_{\alpha}$ is given as in Definition 1 and $z \in\left[\left(X, x_{0}\right)\right.$, $\left.\left(Y, y_{0}\right)\right]$ then

$$
c(G)\left(\tau_{\alpha} \circ \bar{\rho}(z)-\sum_{g \in G} \beta \circ \bar{\alpha}(g * z)\right)=0
$$

in $\left[\left(X, x_{0}\right),\left(W, w_{0}\right)\right]^{\mathrm{ab}}$.

Proof. Define $\zeta=\varepsilon \circ \alpha:\left(Y, y_{0}\right) \rightarrow(L, 1)$ and choose a representative $z: X \rightarrow Y$. Let $M$ be the subgroup of $\operatorname{Hom}\left(\left(X, x_{0}\right),(L, 1)\right)$ 
generated by all the elements of the form $\zeta \circ(g * z)$ and $\zeta \circ(g *\{s \circ \rho \circ z\})$ for all $g \in G$. By Lemma 1 ,

$$
c(G) \sum_{g \in G} \delta \circ \bar{\zeta}(g * z)=c(G) \sum_{g \in G} \delta \circ \bar{\zeta}(g *\{s \circ \rho \circ z\})
$$

in $M^{\mathrm{ab}}$.

From this it follows that

$$
c(G) \sum_{g \in G}[[\bar{\zeta}(g * z)]]=c(G) \sum_{g \in G}[[\bar{\zeta}(g *\{s \circ \rho \circ z\})]]
$$

in $\left[\left(X, x_{0}\right),(L, 1)\right]^{\mathrm{ab}}$, where the inner and outer brackets indicate the two equivalence relations of homotopy and abelianization respectively. The result now follows after applying the isomorphism

$$
\left(\bar{\varepsilon}^{-1}\right)^{\mathrm{ab}}:\left[\left(X, x_{0}\right),(L, 1)\right]^{\mathrm{ab}} \rightarrow\left[\left(X, x_{0}\right),\left(W, w_{0}\right)\right]^{\mathrm{ab}} .
$$

Since the subgroup generated by a single element is abelian, it turns out that, under an additional hypothesis, we can say something about the order of elements in $\left[\left(X, x_{0}\right),\left(W, w_{0}\right)\right]$. The reader can easily furnish the proof of the following by modifying the proof of Theorem 4.

THEOREM 5. Assume the context of Definition 1 and in addition that $[X, Y / G]$ is trivial. Suppose that for some $[f] \in[X, Y]$ and all $g \in G$ the elements $\bar{\alpha}([g * f])$ coincide in $[X, W]$. Then the order of $\bar{\alpha}([f])$ in $[X, W]$ divides $c(G)|G|$.

We next examine the simplest form of our transfer, which occurs when $Y$ is an $H$ space which has the homotopy type of a topological group and $X$ is a co- $H$ space. In this case, the transfer map $\tau$ is a homomorphism from $[X, Y / G]$ to $[X, Y]$. It is in this setting that we feel our use of the term transfer is most justified, since we obtain a transfer map with exactly the same properties as the transfer for singular homology theory, except for the appearance of the factor $c(G)$. This factor can be looked on as the "correction" term that is required because homotopy theory is "not as abelian", in a certain sense (see [R1] for a precise description), as we might like it to be.

THeOREM 6. In addition to the assumptions of Definition 1, assume that $Y=W$ and that $X$ is a co-H space. Then the transfer map 
$\tau:[X, Y / G] \rightarrow[X, Y]$ is a homomorphism with the following properties:

$$
\begin{aligned}
& c(G)\left(\tau \circ \bar{\rho}(z)-\sum_{g \in G} g * z\right)=0 \text { for all } z \in[X, Y] \text {, } \\
& \bar{\rho} \circ \tau(v)=|G| v \quad \text { for all } v \in[X, Y / G] .
\end{aligned}
$$

Proof. Immediate from Theorem 4 and the definitions.

5. The transfer for a generalized homology theory. Next, we develop a transfer for the reduced homology theory associated with any spectrum.

We will follow the definitions and notation for spectra given in [Sw]. Recall that the reduced homology theory, $\tilde{h}_{*}$, associated with a spectrum $E$ is defined by

$$
\tilde{h}_{n}(Y)=\underset{k}{\operatorname{dirlim}} \pi_{k+n}\left(E_{k} \wedge Y, *\right),
$$

where the connecting homomorphisms $\phi_{k}$ are defined by the composition

$$
\pi_{k+n}\left(E_{k} \wedge Y, *\right) \stackrel{\Sigma_{k}}{\rightarrow} \pi_{k+n+1}\left(S^{1} \wedge E_{k} \wedge Y, *\right) \stackrel{l_{k}}{\rightarrow} \pi_{k+1+n}\left(E_{k+1} \wedge Y, *\right) .
$$

It will be convenient in what follows to denote by $\varphi_{k}$ the corresponding homomorphisms used to define $\tilde{h}_{n}(Y / G)$.

Proof of Theorem 1. The proof will be a straightforward "direct limit version" of Theorem 4 . Note that we have not had to make any assumptions involving topological groups. This is because suspending maps is equivalent to mapping into spaces of the form $\Omega S Y$, where $Y$ is a CW complex, and as, is well known, see [St] for example, these spaces are of the homotopy types of topological groups. In what follows, we will extend the group action of $G$ on $Y$ to an action of $G$ on $E_{k} \wedge Y$ in the obvious way.

We begin by taking the spaces $X, Y$ and $W$ in Theorem 4 to be $S^{n+k}, E_{k} \wedge Y$ and $\Omega S\left(E_{k} \wedge Y\right)$, respectively, and the map $\alpha_{k}: E_{k} \wedge$ $Y \rightarrow \Omega S\left(E_{k} \wedge Y\right)$ to be the adjoint of the suspension of the identity map. By Theorem 4, there is a transfer homomorphism

$$
\tau_{\alpha_{k}}: \pi_{n+k}\left(E_{k} \wedge Y / G, *\right) \rightarrow \pi_{n+k}\left(\Omega S\left(E_{k} \wedge Y\right), *\right) .
$$

Let $\operatorname{adj}_{k}: \pi_{n+k}\left(\Omega S\left(E_{k} \wedge Y\right), *\right) \rightarrow \pi_{k+n+1}\left(S^{1} \wedge E_{k} \wedge Y, *\right)$ denote the adjoint homomorphism and define

$$
\tau_{k}=l_{k} \circ \operatorname{adj}_{k} \circ \tau_{\alpha_{k}}: \pi_{n+k}\left(E_{k} \wedge Y / G, *\right) \rightarrow \pi_{k+1+n}\left(E_{k+1} \wedge Y, *\right) .
$$


The theorem now follows immediately from the following easily verified properties:

(a) $\phi_{k+1} \circ \tau_{k}=\tau_{k+1} \circ \varphi_{k}$,

(b) $\rho_{k+1} \circ \tau_{k}(v)=|G| \varphi_{k}(v)$,

(c) $c(G)\left(\tau_{k} \circ \rho_{k}(z)-\sum_{g \in G} \phi_{k}(g * z)\right)=0$.

Using the fact that Eilenberg-Mac Lane spaces have the homotopy types of abelian monoids and the remark following Lemma 1, it can be shown that the coherence number factor $c(G)$ can be dropped in Theorem 1 to obtain a transfer resembling the usual transfer for ordinary homology theory.

\section{Proofs of Theorems (2) and (3).}

Proof of Theorem 2. From Theorem 1, the transfer gives that $c(G) \sum_{g \in G} g * z=0$. Since $\tilde{h}_{n}(X)=\mathbb{Z}$, we have that $\sum_{g \in G} g * z=$ 0 . This can only happen if there is a nontrivial homomorphism from $G$ into the automorphism group $\mathbb{Z}_{2}$ of $\mathbb{Z}$. The result follows immediately.

Proof of Theorem 3. By way of contradiction, assume that the orbit map has a section. The units of order 4 in $\mathbb{Z}_{16}=\{0,1, \ldots, 15\}$ are $3,5,11$ and 13. Since the action is effective, $\rho: \tilde{h}_{n}(W) \rightarrow \tilde{h}_{n}\left(W / \mathbb{Z}_{4}\right)$ cannot be an isomorphism. $\rho$ is a split epimorphism since the orbit map admits a section. Therefore the image of the map in homology induced by the section is a direct summand of $\mathbb{Z}_{16}$. It follows that this image and the image of the transfer map must be trivial. Since $\mathbb{Z}_{4}$ is cyclic, its coherence number is 1 by [R3]. Theorem (1) gives that $\tau \circ \rho(z)=\sum_{g \in G} g * z$ which equals either $8 z$ or $12 z$ depending on which unit of order 4 we take. This contradicts the triviality of $\tau$.

\section{REFERENCES}

[Ba] M. G. Barratt, Spaces of finite characteristic, Quart. J. Math., 11 (1960), 124136.

[Br] G. E. Bredon, Introduction to Compact Transformation Groups, Academic Press, London and New York, (1972).

[C] F. R. Cohen, Orders of Certain Compositions, Seminar on Current Trends in Algebraic Topology (1981), CMS Conference Proceedings, vol. 2 part 1, Amer. Math. Soc., (1982), 289-295.

[KL] D. Kraines and T. Lada, A Counterexample to the Transfer Conjecture, Lecture Notes in Mathematics \#741, Springer-Verlag, New York, Heidelberg, Berlin, (1979), 588-624. 
[R1] W. J. Ralph, Category and group rings in homotopy theory, Trans. Amer. Math. Soc., 299 (1987), 205-223.

[R2] _ An extension of singular homology to Banach algebras, Pacific J. Math., 123, no. 2 (1986), 391-405.

[R3] _ The coherence number of a finite group, J. Algebra, 126 (1989), 61-79.

[St] J. Stasheff, H-Spaces from a Homotopy Point of View, Lecture Notes in Mathematics \#161, Springer-Verlag, New York, Heidelberg, Berlin, (1970).

[Sw] R. M. Switzer, Algebraic Topology-Homotopy and Homology, Springer-Verlag, New York, Heidelberg, Berlin, (1975).

Received March 5, 1990 and in revised form April 25, 1992.

BROCK UNIVERSITY

St. Catherine's, Ontario

CANADA L2S 3A1 



\section{CONTENTS}

G. D. Anderson, M. K. Vamanamurthy, and M. Vuorinen, Inequalities for quasi-

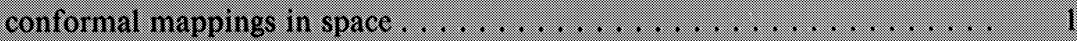

T. Bhattacharya, A nonexistence result for the $n$-Laplacian . . . . . . . . . . . . 19

J. A. Cima, K. Stroethoff, and K. Yale, Bourgain algebras on the unit disk . . . . . 27

J. A. Fridy and C. Orhan, Lacunary statistical convergence . . . . . . . . . . . . . . 43

D. Grenier, On the shape of fundamental domains in $\mathrm{GL}(n, \mathbf{R}) / \mathrm{O}(n) \ldots \ldots . . .53$

B. Jiang and J. Guo, Fixed points of surface diffeomorphisms . . . . . . . . . . . 67

P. Lejarraga, The moduli of rational Weierstrass fibrations over $\mathbf{P}^{\prime}$ : singularities 91

G. J. Martin, On discrete isometry groups of negative curvature . . . . . . . . . . 109

T. Nakashima, Adjoint linear systems on a surface of general type in positive characteristic . . . . . . . . . . . . . . . . . . . . . . . . . 129

B. Ralph, A homotopy transfer for finite group actions $\ldots \ldots \ldots \ldots \ldots \ldots \ldots$

Y. Rong, Maps between Seifert fibered spaces of infinite $\pi_{1} \ldots \ldots \ldots \ldots \ldots, 143$

J.-Y. Shi, Some numeric results on root systems . . . . . . . . . . . . 155

E. Spanier, Singular homology and cohomology with local coefficients and duality

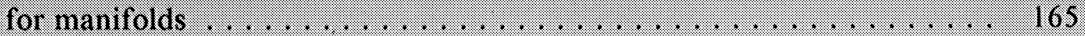




\section{PACIFIC JOURNAL OF MATHEMATICS}

Volume $160 \quad$ No. $1 \quad$ September 1993

Inequalities for quasiconformal mappings in space

GLEN DOUglas ANDERson, MaVina KRISHNa VAMANAMURThy and MATTI VUORINEN

A nonexistence result for the $n$-Laplacian

TILAK BHATTACHARYA

Bourgain algebras on the unit disk

Joseph A. Cima, Karel M. Stroethoff and Keith Yale

Lacunary statistical convergence

JOHN ALBERT FRIDY and CIHAN ORHAN

On the shape of fundamental domains in $\operatorname{GL}(n, \mathbf{R}) / \mathrm{O}(n)$

DOUGLAS MARTIN GRENIER

Fixed points of surface diffeomorphisms

BOJU JIANG and JIANHAN GUO

The moduli of rational Weierstrass fibrations over $\mathbf{P}^{1}$ : singularities

PABLO LEJARRAGA

On discrete isometry groups of negative curvature

GAVEN MARTIN

Adjoint linear systems on a surface of general type in positive characteristic

TOHRU NAKASHIMA

A homotopy transfer for finite group actions

WILLIAM J. RALPH

Maps between Seifert fibered spaces of infinite $\pi_{1}$

YONGWU RONG

Some numeric results on root systems

J. Y. SHI

Singular homology and cohomology with local coefficients and duality for manifolds

EDWIN SPANIER 